\title{
Avoid or engage?
}

Citation for published version (APA):

Simons, L. E., Vlaeyen, J. W. S., Declercq, L., Smith, A. M., Beebe, J., Hogan, M., Li, E., Kronman, C. A., Mahmud, F., Corey, J. R., Sieberg, C. B., \& Ploski, C. (2020). Avoid or engage? Outcomes of graded exposure in youth with chronic pain using a sequential replicated single-case randomized design. Pain, 161(3), 520-531. https://doi.org/10.1097/j.pain.0000000000001735

\section{Document status and date:}

Published: 01/03/2020

DOI:

10.1097/j.pain.0000000000001735

Document Version:

Publisher's PDF, also known as Version of record

\section{Document license:}

Taverne

\section{Please check the document version of this publication:}

- A submitted manuscript is the version of the article upon submission and before peer-review. There can be important differences between the submitted version and the official published version of record.

People interested in the research are advised to contact the author for the final version of the publication, or visit the DOI to the publisher's website.

- The final author version and the galley proof are versions of the publication after peer review.

- The final published version features the final layout of the paper including the volume, issue and page numbers.

Link to publication

\footnotetext{
General rights rights.

- You may freely distribute the URL identifying the publication in the public portal. please follow below link for the End User Agreement:

www.umlib.nl/taverne-license

Take down policy

If you believe that this document breaches copyright please contact us at:

repository@maastrichtuniversity.nl

providing details and we will investigate your claim.
}

Copyright and moral rights for the publications made accessible in the public portal are retained by the authors and/or other copyright owners and it is a condition of accessing publications that users recognise and abide by the legal requirements associated with these

- Users may download and print one copy of any publication from the public portal for the purpose of private study or research.

- You may not further distribute the material or use it for any profit-making activity or commercial gain

If the publication is distributed under the terms of Article $25 \mathrm{fa}$ of the Dutch Copyright Act, indicated by the "Taverne" license above, 


\title{
Avoid or engage? Outcomes of graded exposure in youth with chronic pain using a sequential replicated single-case randomized design
}

\author{
Laura E. Simons $^{\mathrm{a}, \star}$, Johan W.S. Vlaeyen ${ }^{\mathrm{b}}$, Lies Declercq ${ }^{\mathrm{c}}$, Allison M. Smith ${ }^{\mathrm{d}}$, Justin Beebe ${ }^{\mathrm{e}}$, Melinda Hogan ${ }^{f}$,
} Eileen $\mathrm{Li}^{\mathrm{f}}$, Corey A. Kronman ${ }^{\mathrm{a}}$, Farah Mahmud ${ }^{\mathrm{d}}$, Jenelle R. Corey ${ }^{\mathrm{d}}$, Christine B. Sieberg ${ }^{\mathrm{d}, \mathrm{g}}$, Christine Ploski ${ }^{\mathrm{f}}$

\begin{abstract}
Pain-related fear is typically associated with avoidance behavior and pain-related disability in youth with chronic pain. Youth with elevated pain-related fear have attenuated treatment responses; thus, targeted treatment is highly warranted. Evidence supporting graded in vivo exposure treatment (GET) for adults with chronic pain is considerable, but just emerging for youth. The current investigation represents the first sequential replicated and randomized single-case experimental phase design with multiple measures evaluating GET for youth with chronic pain, entitled GET Living. A cohort of 27 youth (81\% female) with mixed chronic pain completed GET Living. For each participant, a no-treatment randomized baseline period was compared with GET Living and 3- and 6-month follow-ups. Daily changes in primary outcomes fear and avoidance and secondary outcomes pain catastrophizing, pain intensity, and pain acceptance were assessed using electronic diaries and subjected to descriptive and model-based inference analyses. Based on individual effect size calculations, a third of participants significantly improved by the end of treatment on fear, avoidance, and pain acceptance. By follow-up, over $80 \%$ of participants had improved across all primary and secondary outcomes. Model-based inference analysis results to examine the series of replicated cases were generally consistent. Improvements during GET Living was superior to the no-treatment randomized baseline period for avoidance, pain acceptance, and pain intensity, whereas fear and pain catastrophizing did not improve. All 5 outcomes emerged as significantly improved at 3-and 6-month followups. The results of this replicated single-case experimental phase design support the effectiveness of graded exposure for youth with chronic pain and elevated pain-related fear avoidance.
\end{abstract}

Keywords: Pediatric pain, Graded exposure in vivo, Pain-related fear, Multilevel modeling, Single-case experimental design

\section{Introduction}

Pain-related fear and avoidance behavior saliently influence pain outcomes, ${ }^{11,31,52-54}$ and both are shown to be associated with disability, depressive symptoms, and school impairment in youth with chronic pain. ${ }^{32,34}$ In the context of pain rehabilitative treatment, decreases in pain-related fear are associated with

\footnotetext{
Sponsorships or competing interests that may be relevant to content are disclosed at the end of this article.

a Department of Anesthesiology, Perioperative and Pain Medicine, Stanford University School of Medicine, Palo Alto, CA, United States, ${ }^{b}$ Research Group Health Psychology, KU Leuven, Belgium \& Experimental Health Psychology, Maastricht University, Maastricht, the Netherlands, ${ }^{\circ}$ Health Psychology, KU Leuven, Leuven, Belgium; Experimental Health Psychology, Maastricht University, The Netherlands, ${ }^{d}$ Department of Anesthesiology, Perioperative and Pain Medicine, Boston Children's Hospital, Boston, MA, United States, ${ }^{\circledR}$ Department of Physical Therapy, Simmons College, Boston, MA, United States, ${ }^{f}$ Department of Physical and Occupational Therapy, Boston Children's Hospital, Boston, MA, United States, ${ }^{g}$ Department of Psychiatry, Boston Children's Hospital and Harvard Medical School, Boston, MA, United States
}

${ }^{*}$ Corresponding author. Address: Stanford University School of Medicine, 1070 Arastradero Rd, Palo Alto, CA 94304, United States. Tel.: (650) 723-6412. E-mail address: lesimons@stanford.edu (L.Simons).

Supplemental digital content is available for this article. Direct URL citations appear in the printed text and are provided in the HTML and PDF versions of this article on the journal's Web site (www.painjournalonline.com).

PAIN 161 (2020) 520-531

(C) 2019 International Association for the Study of Pain

http://dx.doi.org/10.1097/j.pain.0000000000001735 improvements in functional disability and depressive symptoms. ${ }^{29}$ Notably, high levels of pain-related fear at the start of treatment is predictive of attenuated treatment response, suggesting that a more tailored approach that directly targets pain-related fear and avoidance behavior is indicated. ${ }^{29}$ For adults suffering with chronic pain, in vivo exposure treatment (GET) targets pain-related fear through exposing participants to activities previously avoided, ${ }^{47}$ resulting in improved disability and reduced painrelated fear. ${ }^{2}$ Consistent with the Fear Avoidance Model of Chronic Pain, ${ }^{46}$ participants in GET learn that disengagement from safety behaviors does not lead to catastrophic consequences. Thus, their harm beliefs are violated enabling them to recalibrate their expectancies about activity, pain, and injury, ${ }^{12,19,37}$ resulting in decreased fearful cognitions and avoidance behavior.

Typical pain management for youth focuses on pain control through pain management psychology and impairment-based physical therapy and yields modest improvements in functional disability, but no change in pain-related fear. ${ }^{36}$ By contrast, GET targets functional improvement by exposing participants to activities previously avoided due to fear of pain and/or fear of reinjury. There is one randomized controlled trial of graded exposure for adolescents with chronic musculoskeletal pain in the Netherlands that combines GET and physical training, ${ }^{7}$ but outcomes are not yet published.

The current investigation examines GET for youth with chronic pain, entitled GET Living. GET Living represents a significant treatment paradigm shift in pediatric chronic pain 
management by focusing on a key mechanism (pain-related fear and avoidance behavior) rather than on pain itself. GET Living also sets itself apart from previous exposure interventions with an explicit focus on pain willingness and valuebased action derived from Acceptance and Commitment Therapy $^{51}$ (Supplemental Table 1 outlines the ACT-unique elements in bold; available online as supplemental digital content at http://links.Iww.com/PAIN/A898). The specific aim of this study was to evaluate the effectiveness of individually tailored GET Living for youth with chronic pain using a sequential replicated single-case experimental phase design (SCED) with multiple measures. Youth were assessed daily from baseline to end of treatment using electronic diaries to report on the primary outcomes fear and avoidance. The secondary outcomes are pain catastrophizing, pain intensity, and pain acceptance with additional daily assessments for 7 days at 3and 6-month follow-up periods (Fig. 1 depicts assessments and hypothesis). These SCED data were subjected to descriptive and model-based inference analyses. We hypothesized that GET Living would be superior to a no-treatment randomized baseline period. More specifically, we expected (1) improvements on both our primary and secondary outcomes at the end of treatment compared with baseline, and (2) that treatment gains would be maintained at 3- and 6-month follow-ups.

\section{Methods}

\subsection{Study design}

A sequential replicated and randomized SCED with multiple measures was used in this study. In single-case experiments, a subject is observed repeatedly at different levels of at least one independent variable (eg, baseline vs treatment). Each sequential case is considered a replication. The primary treatment outcomes are pain-related fear and avoidance behavior. Secondary outcomes are pain catastrophizing, pain acceptance, and pain severity. These variables are assessed in daily diary form.

\subsection{Participants}

Participants evaluated at the Pain Treatment Service (PTS) inclusive of the Chronic Pain Clinic and Pediatric Headache Program at Boston Children's Hospital $(\mathrm{BCH})$ were recruited from December 2013 to February 2017 to participate in this trial (reg. \# NCT01974791), with data collection (6 months after treatment) completed January 2018. Given that this was a small trial, 2 participants were in active treatment at a time with an ongoing waitlist. Treatment inclusion criteria were: (1) age 8 to 17, (2) elevated pain-related fear at PTS evaluation (score $\geq 40$ on the Fear of Pain Questionnaire $\left[\mathrm{FOPQ}^{34}\right.$ ] or clinician determination if scores were below the cut-off which was further screened by the study team), (3) chronic pain diagnosis, and (4) functional limitations (score $>12$ on the Functional Disability Inventory [FDI ${ }^{16,50}$ or clinician determination if scores were below the cutoff which was further screened by the study team). Participant treatment exclusion criteria were as follows: (1) significant cognitive impairment (eg, intellectual disability), (2) serious psychopathology (eg, active suicidality), (3) acute trauma (eg, spondylolisthesis, disk herniation, fracture, and acute tendonitis), (4) systemic disease in active inflammatory state (eg, rheumatoid arthritis), (5) biomechanical deficit that would limit ability to engage in exposure activities (eg, severe muscle atrophy), and (6) making gains in current physical therapy (PT). Note: clinician determination of eligibility for participants who did not meet FOPQ and FDI selfreport criteria was based on previous clinical experience wherein participants in some cases under report symptoms, but the clinical evaluation suggests the participant is impaired and fear avoidant.

\subsection{Procedures}

Potential enrollment in GET Living was typically presented to the family by a member of the multidisciplinary evaluation team (physician, pain psychologist, or physical therapist). After introducing this treatment option to the family, an eligibility screening form was completed and submitted to the study team. In the case that a participant was referred from a follow-up visit rather than a new multidisciplinary evaluation, the study physical

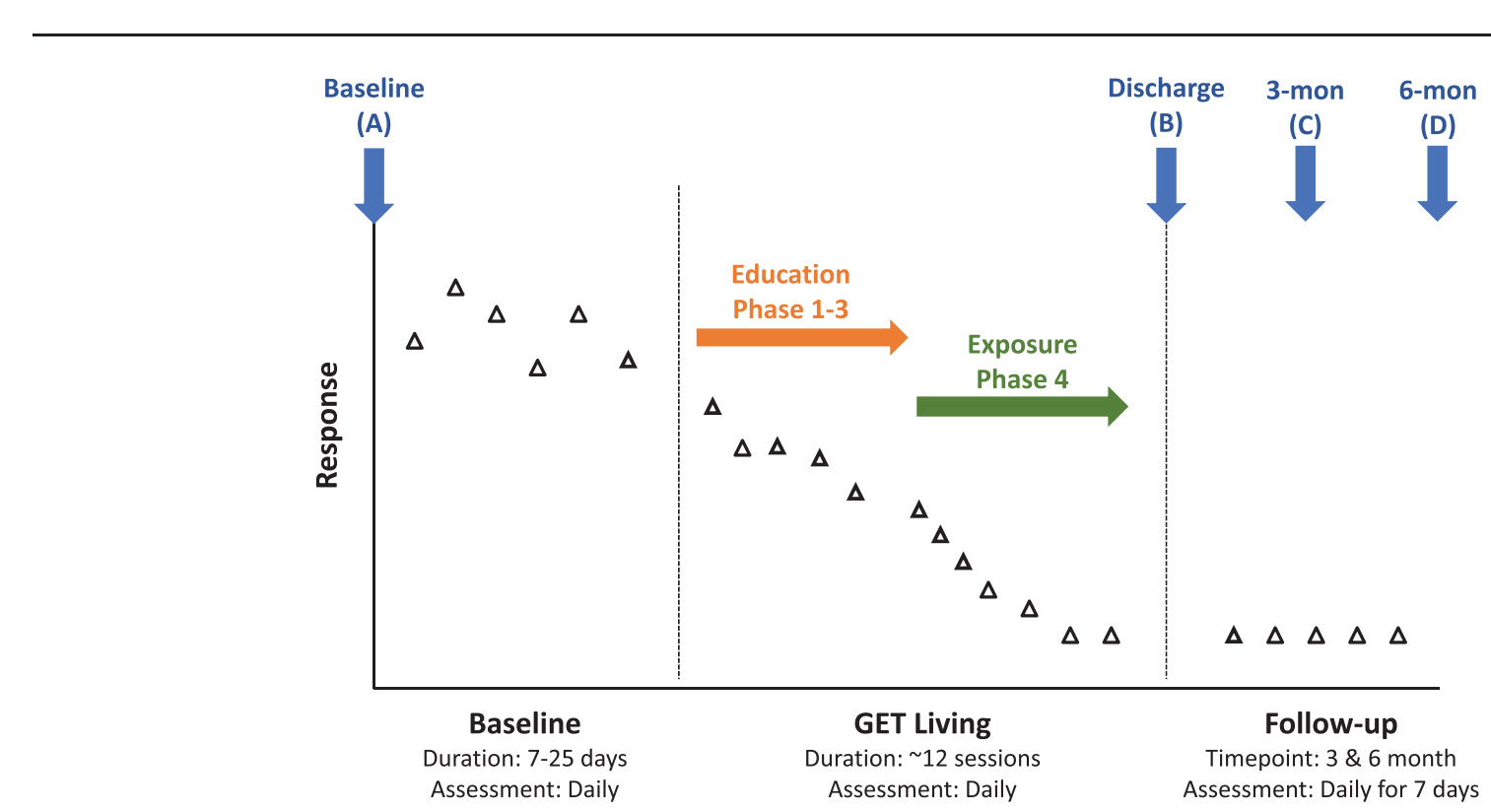

Figure 1. GET Living phases and assessments. Enrollment begins at the baseline assessment with electronic diary surveys occurring daily until discharge. It is hypothesized that daily diary reports will be stable during the randomized baseline period (7-25 days). During education (phase 1-3 of GET Living), it is hypothesized that daily diary reports will remain relatively stable with significant improvements (decreased fear, avoidance, pain, catastrophizing; increased acceptance [increase not depicted for simplicity]) observed during exposure (phase 4 of GET Living). It is hypothesized that 7-day daily diary reports at 3-month and 6-month follow-ups will be stable and reflect sustained improvements in outcomes. 
therapist conducted a PT evaluation to ensure eligibility. After successful screening for eligibility through clinicians and medical record review, participants were scheduled for a baseline visit after any treatment recommendations made at the PTS evaluation had stabilized for a minimum of 2 weeks. At the baseline, participants and a parent completed assent/consent, a battery of measures, and participants were randomly assigned to a baseline period of 7 to 25 days. At the baseline assessment, participants completed the first electronic daily diary in REDCap. For optimal diary engagement with the participant, we (1) selected preferred cellular phone number for text message delivery (emailing a daily link was also a possibility, if preferred), (2) determined preferred time of day, and (3) supervised first completion to answer any potential questions. Participants indicated that the electronic diary delivered through REDCap was easy to complete and brief (1-2 minutes). If diaries were not completed for more than 2 days in a row, the research team would reach out to the participant to prompt completion, and clinicians asked participants about any barriers to diary completion at every treatment session. Following the baseline, participants engaged in GET Living sessions scheduled twice a week for a total of approximately 12 sessions. At the end of treatment, participants and a parent participated in a discharge visit where they completed a battery of measures and participated in a structured exit interview. At both the 3-month and 6-month follow-ups, participants completed a battery of measures and a 7day daily diary. The diary and questionnaire data completed during baseline, treatment/discharge, and 3- and 6-month follow-ups are reported here.

\subsection{Intervention}

\subsubsection{GET Living treatment}

GET Living is a highly structured, protocolized, and individually tailored intervention consisting of individual graded in vivo exposure for the affected child, as well as a parent component to enhance skill acquisition and generalization. An outparticipant treatment team consisting of a psychology clinician (eg, psychologist or supervised psychology trainee) and a physical therapist delivers the intervention. Treatment consists of 5 phases: phase 1-education on fear avoidance, phase 2setting treatment goals, phase 3-build exposure activity ladder, phase 4-exposures, and phase 5-relapse prevention and termination. Phases 1 to 3 are conducted with both clinicians, and child and parent (as developmentally appropriate). Both clinicians are present for these initial/foundational sessions to ensure that the treatment message is consistent from both clinicians to the family. Behavioral exposures begin in phase 4. Exposure activities are typically derived from the Photographs of Daily Activities (PHODA)-Youth (eg, running, lifting objects, and playing soccer). ${ }^{30}$ The clinicians jointly lead a portion of the exposure sessions. During the remaining sessions, the physical therapist leads the exposures while the psychology clinician meets individually with the parent. The decision on whom to include for the different treatment exercises across sessions is negotiated by the clinicians, participant, and parents. Phase 5 wraps up treatment focusing on long-term goal setting and termination. The primary aim of GET Living is the return to valued activities of daily life and restoration of daily functioning (eg, returning to school). If the primary aim is reached before the end of the session series, the clinicians and participant can agree to terminate treatment earlier. Conversely, if additional exposure sessions are deemed therapeutically warranted, the clinician team and participant can agree to add exposure sessions.

\subsubsection{Clinician training}

A session-by-session manual (written by L.E.S. and A.M.S.) was provided to all clinicians. This manual was based on the following books and protocols: "Pain-related fear: exposure-based treatment for chronic pain," 45 "Multimodal CBT treatment for childhood OCD: a combined individual child and family treatment manual," (Piacentini J, Langley A, Roblek T, Chang S, Bergman RL. Multimodal CBT treatment for childhood OCD: a combined individual child and family treatment manual. Unpublished) and "The ACT for teens program." (Greco L. The ACT for Teens Program. Unpublished) In training new psychology clinicians to deliver the treatment, they first observed an experienced provider delivering the treatment to a participant from start to finish (L.E.S. and C.B.S.), then were directly observed delivering treatment to a participant from start to finish. In addition to individual supervision for trainees delivering treatment, the entire GET Living team had weekly conference calls to discuss treatment progress, treatment fidelity, and new referrals. As the trial progressed, we began video-recording sessions ( $n=19)$ for treatment fidelity and future clinician training.

\subsubsection{Treatment fidelity}

Treatment fidelity was coded as presence/absence of concept presented to the participant in clinical notes (for early treatment sessions) or in video recordings ( $n=19$ ). Examples of concepts include present individualized fear avoidance model formulation (phase 1) and identify long-term goals (phase 5). All participant sessions were reviewed for presence/absence of the 35 concepts and independently coded by 2 individuals (See Supplemental Table 1 for list of concepts by phase; available online as supplemental digital content at http://links.lww.com/PAIN/A898). All discrepancies were reviewed, and presence/absence was agreed upon, thus no inter-rater reliability was calculated.

\subsection{Measures}

\subsubsection{Diary}

This electronic daily survey consisted of 12 items selected from the Fear of Pain Questionnaire Fear and Activity Avoidance subscales (FOPQ-C ${ }^{34}$ ), Pain Catastrophizing Scale for Children (PCS-C ${ }^{5}$ ), the Chronic Pain Acceptance Questionnaire-Activity Engagement subscale $\left(\mathrm{CPAQ}^{22}\right)$, and a current pain rating ${ }^{49}$ (See Table 1 for items). All items on the diary were rated on a visual analog scale (VAS) with no numbers shown to the reporter. The VAS values equated to a numerical scale ranging from $0=$ strongly disagree to $10=$ strongly agree, with anchors for pain from $0=$ no pain to $10=$ worst possible pain. Selected items loaded high on the domains of interest in published factor analyses. We conducted internal consistency estimates on all selected item subgroups using a large database of pain participants before implementing the diary items $\left(n=350^{32}\right)$ and in the current sample. Cronbach's alpha was as follows: Two-item FOPQ-Activity Avoidance $(\alpha=0.72$ in database, $\alpha=0.94$ in current sample), three-item FOPQ-Fear $(\alpha=0.76$ in database, $\alpha=0.93$ in current sample), three-item PCS ( $\alpha=0.74$ in database, $\alpha=0.88$ in current sample), and three-item CPAQActivity Engagement $(\alpha=0.72$ in database, $\alpha=0.94$ in current sample). In addition, the daily diary includes an open text box to describe anything exciting or stressful from the past 24 hours. Participants received a text message daily to prompt them to open their electronic daily diary through REDCap and complete it either on their phone or on the computer. Outcomes derived from the daily diary: pain-related fear and activity avoidance (primary); pain catastrophizing, pain acceptance, and pain (secondary). 


\section{Table 1}

\section{Daily diary items.}

Fear

I worry when I am in pain.

I find it difficult to calm my body down when having pain.

Feelings of pain are scary for me.

Avoidance
I put things off because of my pain.
I avoid making plans because of my pain.
Catastrophizing
When I have pain, I keep thinking how much it hurts.
When I have pain, I wonder whether something serious may happen.
When I have pain, it's terrible and I think it's never going to get better.
Acceptance-Activity Engagement
When my pain increases, I can still do things I have to do.
I can do activities well even if I do not control my pain.
I do things that are important and things that are fun although I have chronic
pain.
Pain
On a scale of 0 to 10, tell us how much pain you are feeling right now.

\subsection{Nondaily measures: primary}

\subsubsection{Pain-related fear and avoidance}

The full 24-item version of the FOPQ- $C^{34}$ was administered with total scores calculated for the fear of pain and avoidance of activities subscales. The FOPQ-C has strong psychometric properties in pediatric chronic pain ${ }^{34}$ and headache. ${ }^{33}$ Photograph Series of Daily Activities (PHODA-YE) ${ }^{30}$ is a 50 -item electronic measure with high internal consistency ( $\alpha=0.98$ ) that assesses perceived harm and anticipated pain for the following categories: (1) exercise/sport activities, (2) social/ school activities, (3) upper extremity activities, and (4) activities of daily living. The participant completes the PHODA-YE on a tablet or computer. For each photograph, the participant is asked "On a scale of 0 to 10 , how worried are you that this activity would be harmful to your pain?" and is requested to drag and drop the picture on a worry scale ranging from 0 ("not at all worried") to 10 ("extremely worried") Next, the participant is asked, "On a scale of 0 to 10 , how painful do you think this activity would be?" This question is answered through a slider bar with anchors of 0 ("not at all painful") and 10 ("worst possible pain"). Both the worry and pain scales are in increments of 0 to 10 . When the participant has rated both anticipated worry and pain for the item, they may click to the next activity. Participants cannot advance to the next activity without rating both worry and pain. If an activity does not apply to the participant (eg, the activity is a sport that the participant does not play or the activity is one that the participant does not engage in, such as shaving), the participant may select "This activity does not apply" and move on to the next item. Importantly, we emphasize to the participant that not applicable is not intended for activities they are currently avoiding or fearful of doing. To account for items rated as "Not applicable," subscale scores and total scores are calculated as a mean score of responses provided for worry and pain separately.

\subsection{Nondaily measures: secondary}

\subsubsection{Functioning and pain}

Functional Disability Inventory (FDI $)^{50}$ is a self-report measure of perceived difficulty in performing activities in school, home, physical, and social contexts. The FDI is widely used in pediatric pain research and is recommended as the gold-standard measure of physical functioning for school age children and adolescents for clinical trials in pediatric chronic pain. ${ }^{23}$ The Pediatric Quality of Life Inventory (PedsQL) ${ }^{43,44}$ — school functioning, parent report is a 5 -item scale that investigates how much children have an issue with paying attention in class, forgetting things, keeping up with schoolwork, and missing school due to not feeling well or due to doctor's appointments. Child selfreports and parent proxy reports have been made for this measure, but it has been found that the parent report demonstrates more reliability and validity than the child's. ${ }^{42}$ Higher scores indicate better school functioning. Participants were also asked to provide their average pain rating on a standard 11-point numeric rating scale from 0 ("no pain") to 10 ("most pain possible") ${ }^{48}$ at each assessment point.

\subsubsection{Pain catastrophizing}

The full version of the Pain Catastrophizing Scale, child (PCS-C) ${ }^{5}$ was administered with the total pain catastrophizing score calculated. The internal consistency, factor structure, and validity are well supported. ${ }^{28}$

\subsubsection{Pain acceptance}

The full 20-item Chronic Pain Acceptance Questionnaire (CPAQA) was administered consisting of 2 subscales: activity engagement (11 items) and pain willingness (9 items). As described above, activity engagement reflects the degree of participation in regular daily activities in the presence of pain (eg, "My life is going well, even though I have chronic pain"). Pain willingness reflects the absence of attempts to avoid or control pain (eg, "I avoid putting myself in situations where my pain might increase"reverse keyed). Previous research supports the internal consistency, factor structure, and validity of the adolescent CPAQ. ${ }^{22}$

\subsection{Statistical analyses}

\subsubsection{Daily measurements}

We present both descriptive and model-based inference analyses, based on single-case reporting guidelines ${ }^{20,21,38}$ and recommendations for reporting on a large number of replicated SCED cases. ${ }^{15,24}$ Experts in SCED have developed multilevel modeling for multiple SCEDs (http://52.14.146.253/MultiSCED/). This meta-analysis approach not only combines the participant results, but it also allows for inclusion of moderator variables. ${ }^{1}$

\subsubsection{Descriptive analyses through visual inspection and single-case effect size calculations of single-case experimental phase design}

We have highlighted 2 raw data plots for a treatment responder and nonresponder to demonstrate the individual trajectories that are elucidated through close daily monitoring. Moreover, using the Shiny app for Single-Case Data Analysis (Shiny SCDA; https:// ppw.kuleuven.be/mesrg/software-and-apps/shiny-scda) based on the SCDA Package with $\mathrm{R},{ }^{14}$ we calculated effect sizes for each individual on each outcome comparing phases $(A B, B C, B D$, $A C, A D$ where $A=$ baseline, $B=$ treatment, $C=3$-month followup, $D=6$-month follow-up) using the test statistic, nonoverlap of all pairs. Nonoverlap of all pairs equals the number of comparison pairs showing no overlap, divided by the total number of comparisons. A large effect is indicated by a nonoverlap of all 
pairs value between 0.93 to 1.0 and a medium effect 0.66 to 0.92 . A value less than 0.66 indicates a weak/no effect. ${ }^{26,27}$

\subsubsection{Model-based inference through multilevel modeling of single-case experimental phase design}

When collecting large numbers of individual SCEs, researchers and clinicians have long struggled with how to summarize the results and derive a sensible overall conclusion, free of bias. In this context, Hayes suggested "In order to understand why and how changes happen in an individual, we need to study the processes of change at the level of the individual, and then to gather nomothetic summaries based on collections of such patterns ( $p$. 43)." ${ }^{13}$ Onghena et al. ${ }^{24}$ recently recommended the following: "...if the SCEs are similar enough in design and research focus, then it might also be interesting to combine and compare the results of these multiple participants (page 40)." In tackling this challenge, experts in SCEs have developed multilevel modeling for multiple SCEs. An additional advantage is that such a meta-analysis is not only to combine the participant results, but also to compare the participants by adding a moderator variable. ${ }^{1}$

The data obtained from the randomized SCED used in this study have a hierarchical 2-level structure with observations (level 1) nested within participants (level 2). This nested structure induces dependency within the data: observations vary not only due to random sampling within a participant, but also between different participants. Because of the large number of replicated cases, we used a hierarchical linear model, allowing us to combine all participants' data into one single multilevel model while also taking account both the within- and between-participant dependencies. ${ }^{40,41}$ The within- and between-participant variability are modeled, as well as the overall effects of the treatment across participants. More specifically, the multilevel approach allows us to model a time trend in the treatment phase and provides us with Wald-type $t$-tests and likelihood ratio tests to obtain inference results for both fixed effect and variance component parameters. For conducting the multilevel analysis and for obtaining inference results in $\mathrm{R}^{39}$ the packages Ime $4,{ }^{3}$ ImerTest, ${ }^{18}$ and merTools ${ }^{17}$ were used. The MultiSCED app is now available to assist practitioners in applying multilevel modeling to their SCED data (http://52.14.146.253/MultiSCED/). Detailed description for the 5-phase (baseline, education, exposure, 3-month follow-up, and 6-month follow-up) and 4-phase (baseline, treatment, 3-month follow-up, and 6-month follow-up) models and formulas is in Supplement 1 (available online as supplemental digital content at http://links.Iww.com/PAIN/A898). Correlations among the 5 outcomes are depicted in Table 2.

\subsubsection{Nondaily measures}

We conducted a repeated-measures analysis of variance for each outcome across the 4 timepoints (baseline, discharge, 3-month follow-up, and 6-month follow-up). Mean values, SDs, and effect sizes (partial eta ${ }^{2}$ ) are reported for each outcome.

\subsection{Power and sample considerations}

This study used a sequential replicated randomized SCED with multiple measures, and as such, traditional power and sample size considerations are not applicable. The design of this study was based on previous work done in adults with chronic pain applying the same treatment approach and study design with sample sizes of $6,{ }^{47} 8,{ }^{6}$ and $8 .^{45}$ We aimed to recruit 32 participants to ensure an adequate sample size.

\section{Results}

\subsection{Participant characteristics}

As detailed in the consort diagram (Fig. 2), 73 participants were referred for GET Living treatment. After screening, recruitment, and the baseline period, 33 eligible participants initiated GET Living treatment. Among those who started treatment, 82\% completed GET Living $(n=27)$. As detailed in Table 3, the cohort was predominantly adolescent female and Caucasian. Most common pain diagnosis was chronic neuropathic pain, although a range was represented and participants were typically experiencing pain for least 1 year at the start of treatment.

\subsection{Treatment characteristics}

The baseline period was 14 days on average, with the number of treatment sessions averaging 11.3, just under the projected 12 sessions. Median days enrolled in active treatment were 70 (Table 3).

\subsection{Treatment fidelity}

Adherence to the treatment manual and presentation of the 35 target concepts across the 5 phases of treatment was high. Overall treatment fidelity was $86.7 \%$ (phase 1: $93.9 \%$, phase 2: $84.9 \%$, phase 3: $88.1 \%$, phase $4: 81.7 \%$, phase $5: 84.9 \%$ ).

\subsection{Daily diary completion}

Across participants, $86 \%$ of daily assessments were usable with all participants having $>80 \%$ diary completion across baseline, treatment, and follow-ups. Reasons for unusable data included either missing skipped diaries or completed outside the eligible time interval (greater than \pm 1 day).

\subsection{Descriptive analysis: visual inspection and single-case effect size calculations}

Two cases were selected based on visual inspection and individual effect sizes to highlight raw daily diary data across the 5 outcomes for a treatment responder (Fig. 3) and treatment nonresponder (Fig. 4) and are displayed with the individual effect sizes for each outcome by phase detailed in the Figure legend. Moreover, individual effect sizes for each outcome by phase for each SCED are detailed in Supplemental Table 2 (available online as supplemental digital content at http://links.lww.com/PAIN/A898). At discharge (A-B), a minority of participants reached significant effect size changes ( $n=9$ for fear, $n=10$ for avoidance, $n=4$ for catastrophizing, $n=6$ for pain, $n=9$ for acceptance). At follow-up

\section{Table 2}

Pearson's product-moment correlations for five pain-related outcomes ( $\mathrm{N}=\mathbf{2 2 8 0}$ ).

\begin{tabular}{llllll}
\hline Variables & $\mathbf{1}$ & $\mathbf{2}$ & $\mathbf{3}$ & $\mathbf{4}$ & $\mathbf{5}$ \\
\hline 1. Fear & - & $0.61^{*}$ & $-0.35^{*}$ & $0.43^{*}$ & $0.84^{*}$ \\
\hline 2. Avoidance & & - & $-0.52^{*}$ & $0.30^{*}$ & $0.53^{*}$ \\
\hline 3. Acceptance & & & - & $-0.22^{*}$ & $-0.40^{\star}$ \\
\hline 4. Pain & & - & $0.47^{*}$ \\
\hline 5. Catastrophizing & & & - & -
\end{tabular}


Table 3

Patient demographic and medical characteristics $(n=27)$.

\begin{tabular}{|c|c|c|c|}
\hline Variable & Range & Mean (SD) & Frequency \% (n) \\
\hline \multicolumn{4}{|l|}{ Demographic characteristics } \\
\hline Age (y) & $8-17$ & $13.5(2.6)$ & \\
\hline Female & & & $81(22)$ \\
\hline \multicolumn{4}{|l|}{ Ethnicity } \\
\hline Caucasian & & & $89(24)$ \\
\hline African American & & & $4(1)$ \\
\hline Biracial & & & $7(2)$ \\
\hline \multicolumn{4}{|l|}{ Parent Marital status } \\
\hline Married & & & $85(23)$ \\
\hline Single & & & $4(1)$ \\
\hline Divorced/separated & & & $11(3)$ \\
\hline \multicolumn{4}{|l|}{ Pain diagnosis } \\
\hline Musculoskeletal & & & $37(10)$ \\
\hline Neuropathic & & & $41(11)$ \\
\hline Abdominal & & & $15(4)$ \\
\hline Headache & & & $7(2)$ \\
\hline Duration of pain (mo) & $2-103$ & Median $=12$ & \\
\hline 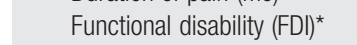 & $2-47$ & $24.9(10.3)$ & \\
\hline Fear of pain (FOPQ-Total) ${ }^{\star}$ & $9-82$ & $50.6(20.8)$ & \\
\hline \multicolumn{2}{|l|}{ Variable } & Range & Median \\
\hline \multicolumn{4}{|l|}{ Treatment characteristics } \\
\hline \multicolumn{2}{|l|}{ Duration of baseline (d) } & $7-25$ & 14 \\
\hline \multirow{2}{*}{\multicolumn{2}{|c|}{$\begin{array}{c}\text { Total no. of treatment sessions } \\
\text { No. of exposures sessions }\end{array}$}} & $6-15$ & 12 \\
\hline & & $1-9$ & 5 \\
\hline \multicolumn{2}{|l|}{ No. of treatment days } & $40-197$ & 70 \\
\hline
\end{tabular}

* As observed from the ranges, a few patients were deemed eligible based on clinician determination and study team screening, rather than score criteria.

(A-C, A-D, B-C, B-D), most effects were large with the majority evidencing change $(n=24$ for fear, $n=22$ for avoidance, $n=23$ for catastrophizing, $n=22$ for pain, $n=22$ for acceptance).

\subsection{Model-based inference: multilevel modeling of daily diary outcomes}

Likelihood ratio tests were used to verify whether the two-level model extended with slopes for the education and exposure phases (Equation 3) provided a significantly better fit than the model without the slopes (Equations 1 and 2). These tests were conducted for each of the 5 outcomes separately and showed that the model including the slope did indeed result in a better fit $\left(\chi_{\text {Fear }}^{2}(15)=1061.4, P<.001\right.$;

$\chi_{\text {Avoidance }}^{2}(15)=822.15, \quad P<0.01$;

$\chi_{\text {Acceptance }}^{2}(15)=376.85, P<0.01$;

$\chi_{\text {Catastrophizing }}^{2}(15)=831.47, P<0.01$;

$\left.\chi_{\text {Pain }}^{2}(15)=243.82, P<0.01\right)$. The results of the estimations for the model (Equation 3) are shown in Table 4. To complement the numerical results from Table 4, the estimated individual regression lines of the participants and the estimated average trajectory are shown in Figures 5 and $\mathbf{6}$ for the primary outcomes of fear and avoidance and Supplementary Figures 1-3 (available online as supplemental digital content at http:// links.Iww.com/PAIN/A898) for secondary outcomes of acceptance, catastrophizing, and pain. The individual (colored) lines were estimated by sampling from the distribution of the estimated fixed and random effects and then estimating the fitted value across that distribution (Knowles and Frederick, 2016). The average trajectories shown in black are based on the fixed-effects estimates from Table 4.

\subsection{Interpretation of models and rate of change}

The results displayed in Table 4 and Figures 5 and 6, and Supplementary Figures 1-3 (available online as supplemental digital content at http://links.Iww.com/PAIN/A898), reveal that the baseline level is on average around 5 to 6 on the ten-point
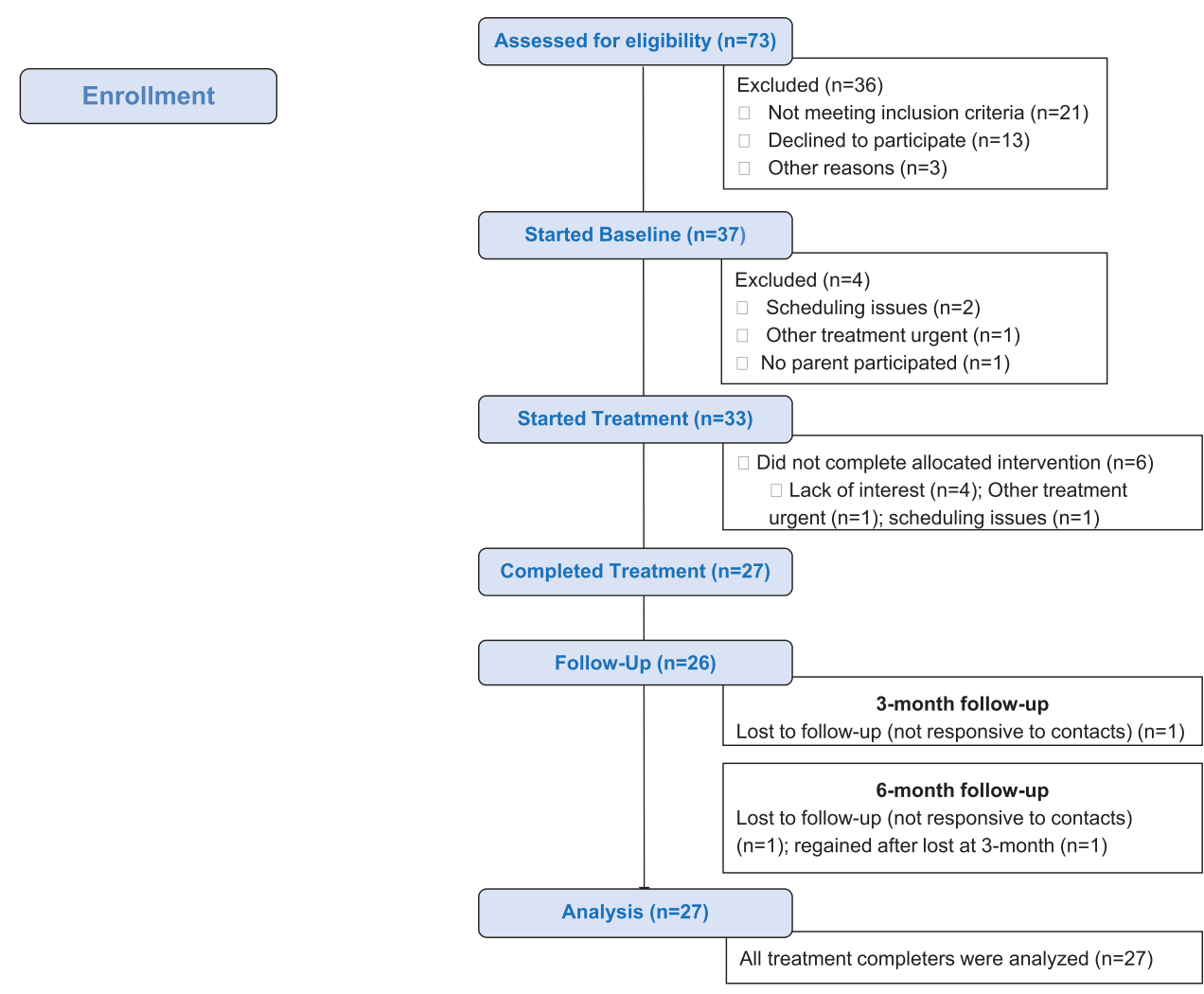

Figure 2. CONSORT flowchart of enrollment. 


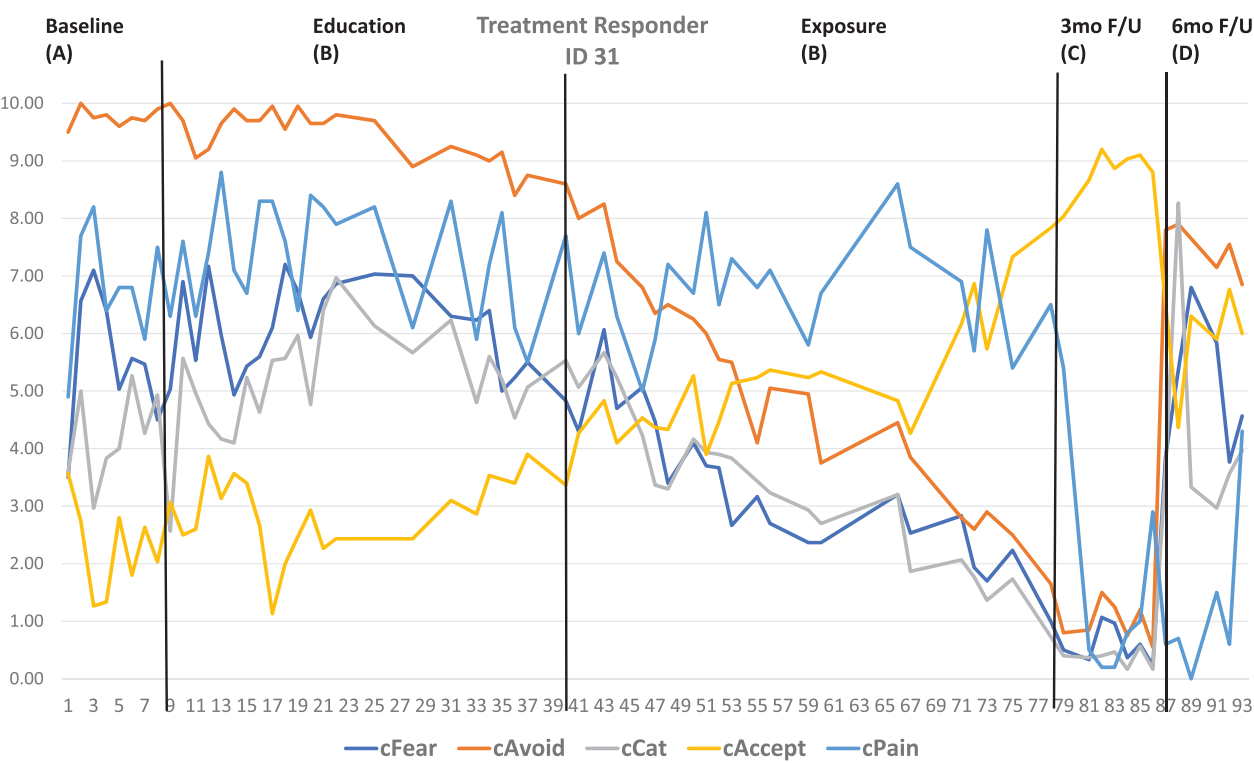

Figure 3. Raw data from treatment responder. The raw daily diary data across outcomes and timepoints for a treatment responder is depicted for visual inspection and descriptive analysis. Through visual inspection, the baseline values across outcomes are relatively stable with modest improvements observed during the education phase of treatment. The slope of improvement was much steeper during the exposure phase, with stable improvement at 3-month follow-up, with reemergence of pain-related distress at 6-month follow-up. Comparing baseline with end of treatment (means phase A minus means phase B), fear = 1.04 (0.66), avoidance $=2.86(0.90)$, and acceptance $=1.78(0.84)$ improved with medium individual effects. Comparing baseline with 3-month follow-up (means phase A minus means phase C), fear $=5.01(1.0)$, avoidance $=8.75(1.0)$, catastrophizing $=3.84(1.0)$, pain $=5.88(1.0)$, and acceptance $=6.57(1.0)$ improved with large individual effects. Interestingly at 6-month follow-up when compared baseline (means phase A = minus means phase $D)$, avoidance $=2.29(1.0)$, pain $=5.53$ (1.0), and acceptance $=3.60$ (1.0) remained improved with large individual effects but fear and catastrophizing have re-emerged. Detailed individual data combining visual inspection and effect size calculation affords a focused view of the data that can immediately inform treatment delivery for this specific participant.

scale for all outcomes. At the start of the education phase, there is on average no immediate effect for 4 of the 5 outcomes. Only for pain catastrophizing, an increase of 0.43 points $(P<0.05)$ on average was detected at the start of the education phase. For pain avoidance and for the pain severity, the effect of treatment manifests itself in the significant negative slopes: on average, the pain avoidance score decreases by $0.27(P<0.05)$ points every 10 days during the education phase and the pain severity decreases by $0.38(P<0.05)$ points every 10 days during the exposure phase. The pain outcome decreases significantly by

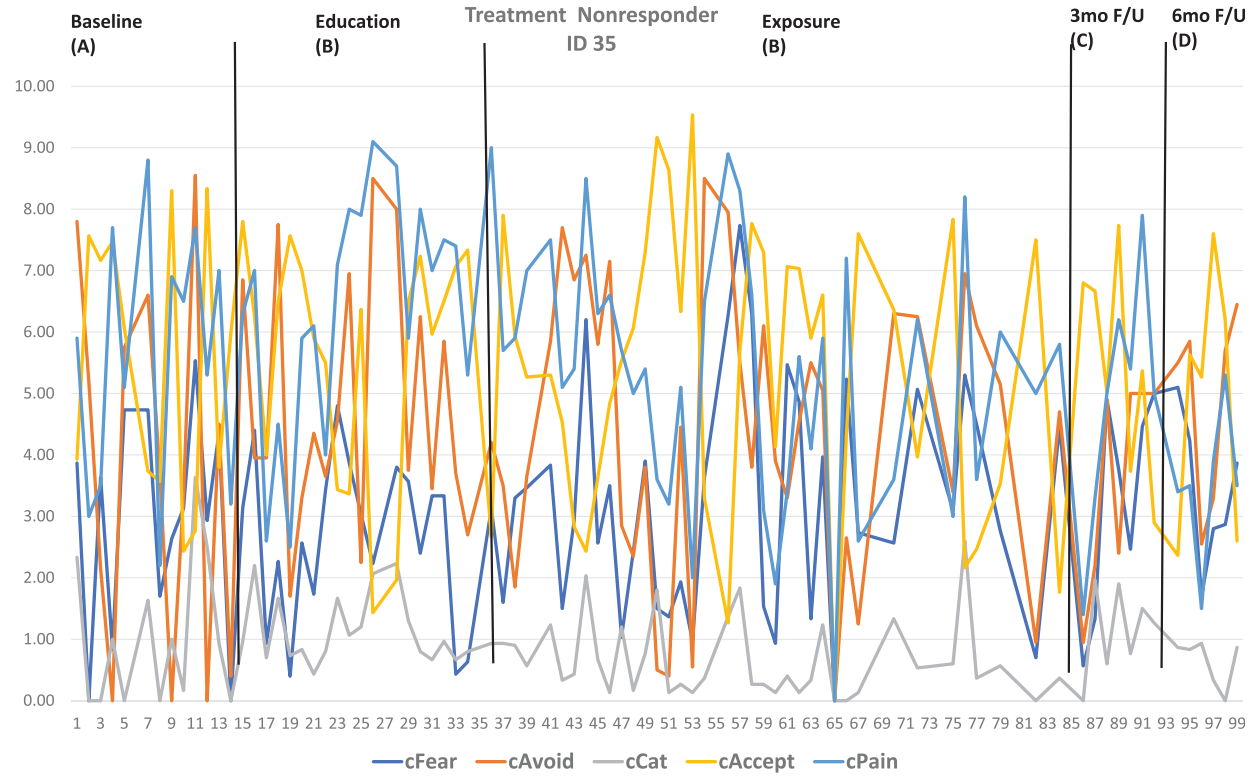

Figure 4. Raw data from treatment nonresponder. The raw daily diary data across outcomes and timepoints for a treatment nonresponder are depicted for visual inspection and descriptive analysis. Through visual inspection, no improvements are observed across outcomes. Through individual effect size calculations, when comparing baseline with 6-month follow-up (means phase A minus means phase D), pain $=2.22(0.78)$ improved with medium individual effects. No other outcomes or significant effects were observed. Detailed individual data combining visual inspection and effect size calculation afford a focused view of the data that can immediately inform treatment delivery for this specific participant. 
0.38 points $(P<0.05)$ every 10 days. No significant changes in pain-related fear, pain catastrophizing, or pain acceptance were observed.

When examining the multilevel model with treatment combined (education + exposure), acceptance emerged as statistically significant during treatment (Table 5). This added finding is likely due to increased power when combining treatment phases as increased acceptance is visually evident in Supplementary Figure 1 (available online as supplemental digital content at http://links.Iww.com/PAIN/A898).

\subsection{Outcomes at 3- and 6-month follow-ups}

For the follow-up phases, an identical pattern of the fixed effects was observed across all outcomes expected to decrease with treatment (fear, avoidance, catastrophizing, and pain): the average score in the 3-month follow-up phase and the 6-month follow-up phase is lower compared with the baseline phase. This suggests that the therapeutic effect of treatment continues over time.

\subsection{Moderator analysis by pain diagnosis}

Given the diversity of pain diagnoses in this cohort, we examined this participant characteristic as a potential moderator of treatment outcome. Participants' pain diagnoses were classified into 4 categories (musculoskeletal, neuropathic, abdominal, and headache) and can thus be included in the model (Equations 3 and 4) as 3 dummy variables that equal 1 if an observation belongs to a participant who falls in the category denoted by the dummy and 0 otherwise, with the fourth category being the reference category. Again, likelihood ratio tests were used to verify whether including pain diagnosis as a moderator in the model (Equations 3 and 4) lead to a significantly better fit with smaller between-participant variability in the regression coefficients. These tests showed that this was not the case for any of the outcomes $\left(\chi_{\text {Acceptance }}^{2}(18)=27.23, P=0.07\right.$; $\chi_{\text {Avoidance }}^{2}(18)=20.506, P=0.31$;

$\chi_{\text {Catastrophizing }}^{2}(18)=25.15, P=0.12$;

$\left.\chi_{\text {Fear }}^{2}(18)=18.91, P=0.40 ; \chi_{\text {Pain }}^{2}(18)=24.46, P=0.14\right)$.

\subsection{Nondaily outcomes}

\subsubsection{Primary outcomes}

Youth reported significant improvements in all primary outcome metrics (Table 6). Large effects were observed for decreased activity avoidance, perceived harm, and anticipated pain, with improvements at discharge that maintained at follow-up. Medium effects were observed for decreased fear of pain, with significant improvements observed at follow-up.

\subsubsection{Secondary outcomes}

Youth reported significant improvements in all secondary outcome metrics. Large effects were observed for decreased functional disability and improved school functioning. Medium effects were observed for pain, pain catastrophizing, pain acceptance (pain willingness, activity engagement), and school attendance. Of note, pain willingness improvements were delayed and observed at follow-up.

\section{Discussion}

The current report represents the first study examining the outcomes of graded exposure in vivo treatment for pediatric participants with chronic pain using electronic daily diary methodology, demonstrating positive effects on all of the pain-related outcomes. Within the context of a series of rigorous replicated and randomized SCEDs, participants completed daily assessments of pain-related fear, avoidance, acceptance, catastrophizing, and pain intensity. Given the large number of replicated cases, in addition to descriptive analyses highlighting treatment responder/nonresponder cases and calculating individual effects sizes for each of the 5 outcomes, we applied innovative multilevel modeling to our SCED data. This reflects one of the first applications of this methodology to daily assessment of biobehavioral treatment outcomes in any health condition. Applied to SCED data, multilevel modeling offers a flexible method and enabled us to model data across participants in one comprehensive model. ${ }^{25,26}$ With statistical tools (MultiSCED) now available to assist practitioners in applying multilevel modeling to

\section{Table 4}

Multilevel model estimations for primary and secondary outcomes from baseline to education, exposure, 3-month follow-up, and 6-month follow-up.

\begin{tabular}{|c|c|c|c|c|c|}
\hline & \multicolumn{2}{|c|}{ Primary outcomes } & \multicolumn{3}{|c|}{ Secondary outcomes } \\
\hline & Fear & Avoidance & Catastrophizing & Acceptance & Pain \\
\hline \multicolumn{6}{|l|}{ Fixed effects } \\
\hline Intercept $\left(\gamma_{00}\right)$ & $6.12(0.48)^{\star \star \star}$ & $6.26(0.51)^{\star \star \star}$ & $5.35(0.52)^{\star \star \star}$ & $5.04(0.42)^{\star \star \star}$ & $5.99(0.50)^{\star \star \star}$ \\
\hline Education $\left(\gamma_{10}\right)$ & $0.39(0.27)$ & $0.30(0.28)$ & $0.43(0.18)^{\star}$ & $-0.17(0.21)$ & $-0.08(0.14)$ \\
\hline Education slope $\left(\gamma_{20}\right)$ & $-0.03(0.06)$ & $-0.27(0.12)^{\star}$ & $-0.05(0.09)$ & $-0.04(0.06)$ & $-0.04(0.08)$ \\
\hline Exposure $\left(\gamma_{30}\right)$ & $0.10(0.26)$ & $-0.45(0.40)$ & $0.16(0.27)$ & $-0.10(0.34)$ & $-0.10(0.29)$ \\
\hline Exposure slope $\left(\gamma_{40}\right)$ & $-0.20(0.21)$ & $-0.39(0.23)$ & $-0.21(0.21)$ & $0.33(0.20)$ & $-0.38(0.18)^{\star}$ \\
\hline 3 -month follow-up $\left(\gamma_{50}\right)$ & $-2.37(0.50)^{\star \star \star}$ & $-3.09(0.69)^{\star \star \star}$ & $-2.05(0.53)^{\star \star \star}$ & $2.04(0.41)^{\star \star \star}$ & $-2.25(0.53)^{\star \star \star}$ \\
\hline 6-month follow-up $\left(\gamma_{60}\right)$ & $-1.90(0.51)^{\star \star \star}$ & $-2.61(0.63)^{\star \star \star}$ & $-1.57(0.47)^{\star \star}$ & $1.92(0.42)^{\star \star \star}$ & $-2.12(0.55)^{\star \star \star}$ \\
\hline \multicolumn{6}{|l|}{ Variance between patients } \\
\hline Intercept & $6.03^{\star \star \star}$ & $7.10^{\star \star \star}$ & $7.23^{\star \star \star}$ & $4.70^{\star \star \star}$ & $6.67^{\star \star \star}$ \\
\hline Education & $1.79^{\star \star \star}$ & $1.86^{\star \star \star}$ & $0.72^{\star \star \star}$ & $1.00^{\star \star \star}$ & 0.22 \\
\hline Education slope & $0.06^{\star \star \star}$ & $0.29^{\star \star \star}$ & $0.14^{\star \star \star}$ & $0.04^{\star \star \star}$ & $0.08^{*}$ \\
\hline Exposure & $1.56^{\star \star *}$ & $3.91^{\star \star \star}$ & $1.85^{\star \star \star}$ & $2.93^{\star \star \star}$ & $1.85^{\star \star \star}$ \\
\hline Exposure slope & $1.13^{\star \star \star}$ & $1.29^{\star \star \star}$ & $1.14^{\star \star \star}$ & $1.03^{\star \star \star}$ & $0.72^{\star \star \star}$ \\
\hline 3-month follow-up & $6.35^{\star \star \star}$ & $12.51^{\star \star \star}$ & $7.22^{\star \star \star}$ & $4.21^{\star \star \star}$ & $7.19^{\star \star \star}$ \\
\hline 6-month follow-up & $6.47^{\star \star \star}$ & $10.02^{\star \star \star}$ & $5.68^{\star \star \star}$ & $4.30^{\star \star \star}$ & $7.65^{\star \star \star}$ \\
\hline
\end{tabular}

${ }^{\star} P<0.05,{ }^{\star \star} P<0.01,{ }^{\star \star \star} P<0.001$. $P$-values based on Wald-type $t$-tests with Kenward-Roger's degrees of freedom approximation for fixed effects and on likelihood ratio tests for variance components. The intercept $\gamma_{00}$ is the average baseline level. The parameters $\gamma_{10}, \gamma_{30}, \gamma_{50}$, and $\gamma_{60}$ indicate the average change in level at the start of, respectively, the exposure, education, 3-month and 6-month follow-up phases compared with the average level at the baseline phase. The parameters $\gamma_{20}$ and $\gamma_{40}$ indicate the average slope in the education and exposure phases, respectively. SEs are given in parentheses. 

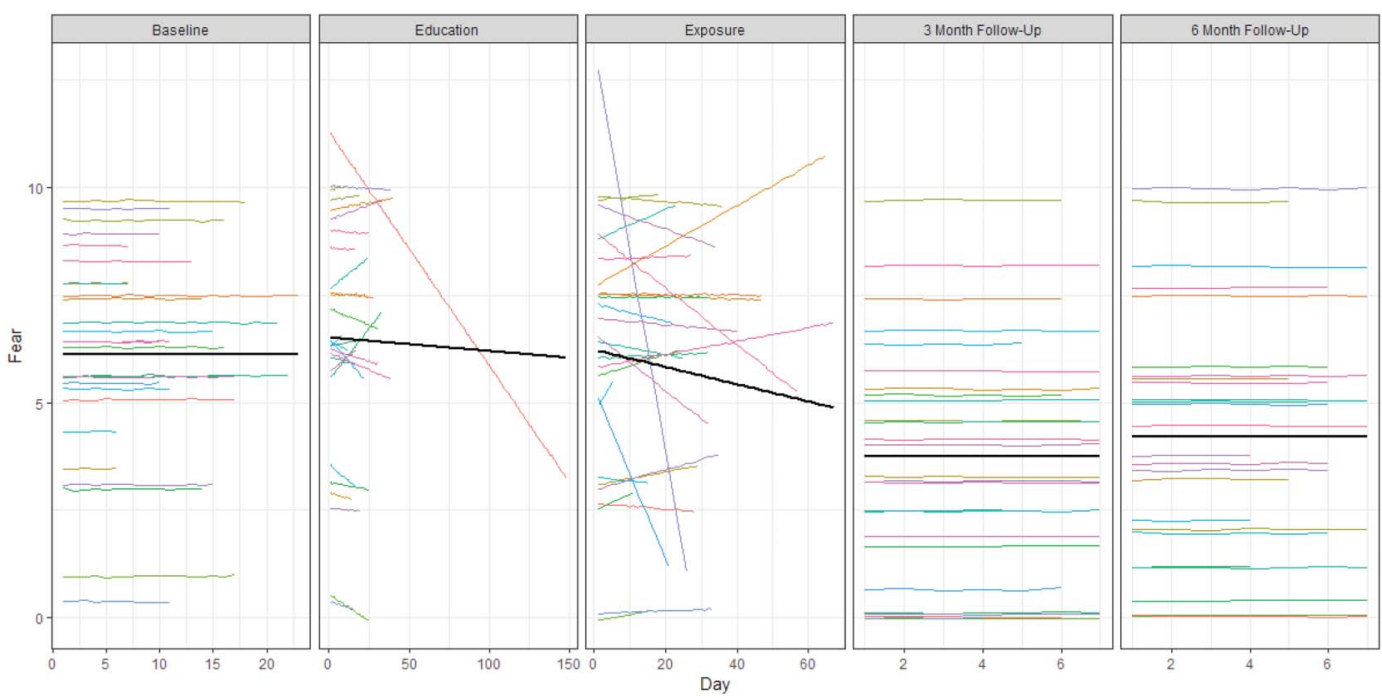

Figure 5. Estimated regression lines based on the multilevel model for fear. Individual participant trajectories are shown in color $(\mathrm{N}=27)$; the overall average trajectory across participants is shown in black.

SCED data, it is likely to become a more common practice for evaluating large numbers of replicated cases. ${ }^{1}$

The results partially supported our hypotheses. From the descriptive analyses of individual effect sizes, a third of participants significantly improved by the end of treatment on fear, avoidance, and pain acceptance. By follow-up, over $80 \%$ of participants had improved across all primary and secondary outcomes. Modelbased inference analysis results of the series of replicated cases were generally consistent with the individual outcomes. For our primary outcomes, we observed significant decreases in pain-related avoidance during treatment that was maintained at 3-month and 6month follow-ups. For pain-related fear, significant decreases were only observed from the 3-month follow-up assessment on, with improvements maintained at 6-month follow-up, indicating a delayed treatment effect. For secondary outcomes, pain acceptance and pain intensity significantly improved during treatment with this continuing at both follow-up timepoints. By contrast, pain catastrophizing also showed delayed effects, with improvements at 3-month follow-up, with improvements maintained at 6-month follow-up. This is further underscored with medium to large improvements in questionnaire versions across primary and secondary outcomes. Furthermore, the large effects detected for decreased functional disability and improved school functioning support the positive impact of GET Living on daily life functioning. Although we anticipated significant improvement across all outcomes by the end of treatment that continued at follow-up, the pattern of results provides conclusions that deserve further inquiry. Given the behavior measures of avoidance and activity engagement (acceptance) and cognitive measures of fear and catastrophizing (perhaps more aptly described as pain-related worry ${ }^{19}$ ), these results suggest a potential behavior -> cognition "order of operations" in relation to treatment response for this approach among youth with chronic pain. The time between discharge and follow-up assessments allows for repetition and generalization of extinction of pain-related fear, and repetition is what shifts rapid behavior change into a shift in beliefs later on. Moreover, the focus of GET Living was on activity and exposure rather than on changing cognitions. This is consistent with inhibitory learning and ACT approaches that focus on behavioral experience
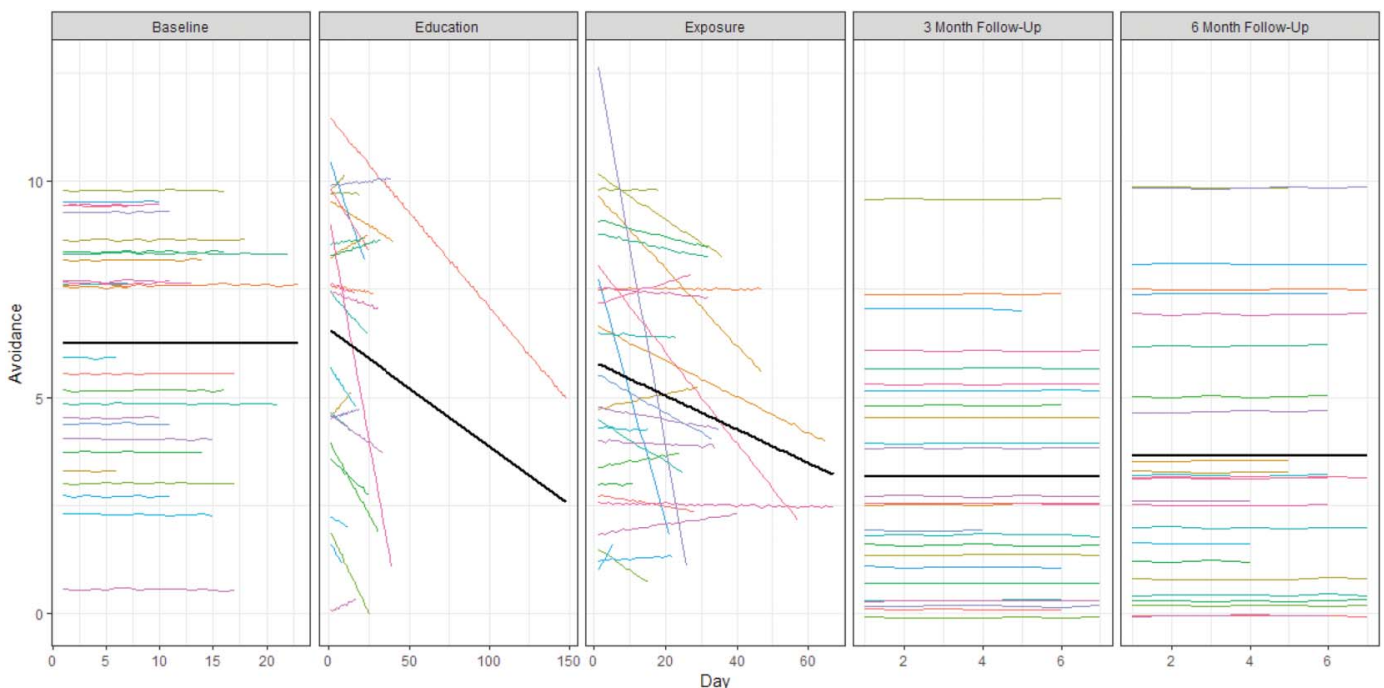

Figure 6. Estimated regression lines based on the multilevel model for avoidance. Individual participant trajectories are shown in color ( $N=27)$; the overall average trajectory across participants is shown in black. 
Table 5

Multilevel model estimations for primary and secondary outcomes from baseline to treatment, 3-month follow-up, and 6-month follow-up.

\begin{tabular}{|c|c|c|c|c|c|}
\hline & \multicolumn{2}{|c|}{ Primary outcomes } & \multicolumn{3}{|c|}{ Secondary outcomes } \\
\hline & Fear & Avoidance & Catastrophizing & Acceptance & Pain \\
\hline \multicolumn{6}{|l|}{ Fixed effects } \\
\hline Intercept $\left(\gamma_{00}\right)$ & $6.12(0.47)^{\star \star \star}$ & $6.27(0.51)^{\star \star \star}$ & $5.34(0.52)^{\star * *}$ & $5.04(0.42)^{\star \star \star}$ & $5.98(0.49)^{\star \star \star}$ \\
\hline Treatment $\left(\gamma_{10}\right)$ & $0.52(0.29)$ & $0.28(0.36)$ & $0.53(0.21)^{\star}$ & $-0.43(0.28)$ & $0.10(0.17)$ \\
\hline Treatment slope $\left(\gamma_{20}\right)$ & $-0.10(0.06)$ & $-0.23(0.07)^{\star \star}$ & $-0.10(0.06)$ & $0.14(0.05)^{\star}$ & $-0.01(0.01)^{\star}$ \\
\hline 3-month follow-up $\left(\gamma_{30}\right)$ & $-2.37(0.50)^{\star \star \star}$ & $-3.10(0.69)^{\star \star \star}$ & $-2.06(0.53)^{\star \star \star}$ & $2.08(0.40)^{\star \star \star}$ & $-2.19(0.52)^{\star \star \star}$ \\
\hline 6-month follow-up $\left(\gamma_{40}\right)$ & $-1.91(0.51)^{\star \star}$ & $-2.59(0.63)^{\star \star \star}$ & $-1.58(0.48)^{\star \star}$ & $1.94(0.42)^{\star \star \star}$ & $-2.06(0.55)^{\star \star}$ \\
\hline \multicolumn{6}{|l|}{ Variance between patients } \\
\hline Intercept & $6.01^{\star * \star}$ & $7.09^{\star \star \star}$ & $7.21^{\star \star \star}$ & $4.66^{\star \star \star}$ & $6.62^{\star \star \star}$ \\
\hline Treatment & $2.04^{\star \star \star}$ & $3.30^{\star \star \star}$ & $1.01^{\star \star \star}$ & $1.85^{\star \star \star}$ & $0.48^{\star \star \star}$ \\
\hline Treatment slope & $0.10^{\star \star \star}$ & $0.13^{\star \star \star}$ & $0.09^{\star \star \star}$ & $0.07^{\star \star \star}$ & $0.09^{\star \star \star}$ \\
\hline 3-month follow-up & $6.40^{\star \star \star}$ & $12.52^{\star \star \star}$ & $7.19^{\star \star \star}$ & $4.02^{\star \star \star}$ & $7.00^{\star \star \star}$ \\
\hline 6-month follow-up & $6.34^{\star \star \star}$ & $10.03^{\star \star \star}$ & $5.75^{\star \star \star}$ & $4.23^{\star \star \star}$ & $7.76^{\star \star \star}$ \\
\hline
\end{tabular}

${ }^{\star} P<0.05,{ }^{\star \star} P<0.01,{ }^{\star \star \star} P<0.001$. $P$-values based on Wald-type $t$-tests with Kenward-Roger's degrees of freedom approximation for fixed effects and on likelihood ratio tests for variance components. The intercept $\gamma_{00}$ is the average baseline level. The parameters $\gamma_{10}, \gamma_{30}$, and $\gamma_{40}$ indicate the average change in level at the start of, respectively, the treatment, 3-month and 6-month follow-up phases compared with the average level at the baseline phase. The parameter $\gamma_{20}$ is the average slope in the treatment phase.

providing the most salient learning and ultimately leading to changes in cognitions. Thus, the current order of operations may actually be most consistent with the targeted mechanisms in this treatment approach. The concept of "order of operations" has garnered some attention, although typically examined in relation to predicting better/ worse outcomes in adult pain ${ }^{4}$ or in relation to improvements in pain and function. ${ }^{25}$ The questionnaire data support our daily diary findings with immediate improvements observed for avoidance, pain, and activity engagement (acceptance) at discharge and fear significantly improved at follow-up.

With regards to pain intensity, although some studies describe delayed effects for improvements in pain, ${ }^{8,35,45}$ others have observed a more immediate effect. ${ }^{25}$ In our study, we observed improvements in pain severity during the exposure phase of treatment, rather than only at follow-up. It may be that changes in behavior are more closely related to changes in pain severity rather than changes in cognitions. Evaluating these outcomes from a daily perspective provides the opportunity to evaluate the slope of change and obtain a more nuanced approach to tracking progress and to understanding how these different outcomes evolve over the course of treatment. This level of detail would be missed if only pre-post questionnaire data were available from these participants.

Another key marker of progress is the degree of change observed in outcomes within windows of time. Results suggest the most marked improvement was in avoidance $(0.23$ points on a 10-point scale every 10 days), which is highly consistent with the primary target of the GET Living intervention. The treatment centers on identifying worrisome and currently avoided activities and designing individualized exposure exercises to approach these valued activities. Latter changes in cognitive outcomes may be driven through new behaviors and subsequent experiences that trigger violations in catastrophic expectancies as new learning occurs. Forming new inhibitory associations without the occurrence of the feared outcome is also key to extinction, the experimental analogue to graded exposure. In future research, it would be interesting to examine how improvements in avoidance behavior more specifically mediate subsequent changes in painrelated worries (fears and catastrophic cognitions).

\section{Table 6}

Mean values across for primary and secondary outcomes.

\begin{tabular}{|c|c|c|c|c|c|c|}
\hline & Baseline, M (SD) & Discharge, M (SD) & 3-month FU, M (SD) & 6-month FU, M (SD) & Greenhouse-Geisser $\mathrm{F}$ & Partial Eta ${ }^{2}$ \\
\hline \multicolumn{7}{|l|}{ Primary } \\
\hline FOPQ-fear $(n=25)$ & $28.6(11.9)$ & $21.6(14.4)$ & $18.0(14.1)^{\mathrm{a}}$ & $17.6(13.1)^{\mathrm{b}}$ & 9.56 & $0.285^{*}$ \\
\hline FOPQ-avoidance $(n=25)$ & $24.2(9.2)$ & $14.9(11.7)^{\mathrm{C}}$ & $12.6(9.9)^{\mathrm{a}}$ & $12.4(9.7)^{\mathrm{b}}$ & 20.94 & $0.466^{*}$ \\
\hline PHODA-PH $(n=24)$ & $5.01(2.34)$ & $2.83(2.45)^{\mathrm{C}}$ & $1.89(2.31)^{\mathrm{a}, \mathrm{d}}$ & $1.94(1.92)^{\mathrm{b}, \mathrm{e}}$ & 22.20 & $0.491^{*}$ \\
\hline PHODA-AP $(n=24)$ & 5.09 (1.92) & $2.82(2.40)^{C}$ & $1.91(2.24)^{\mathrm{a}, \mathrm{d}}$ & $2.04(2.13)^{b}$ & 26.21 & $0.533^{*}$ \\
\hline \multicolumn{7}{|l|}{ Secondary } \\
\hline Functional disability $(n=24)$ & $25.0(10.8)$ & $15.3(11.9)^{C}$ & $10.4(11.2)^{\mathrm{a}, \mathrm{d}}$ & $11.0(10.8)^{b}$ & 26.38 & $0.534^{*}$ \\
\hline School functioning $(n=24)$ & $45.5(20.9)$ & $58.1(17.8)^{C}$ & $67.1(15.1)^{\mathrm{a}, \mathrm{d}}$ & $68.9(20.9)^{\mathrm{b}, \mathrm{e}}$ & 19.30 & $0.456^{\star}$ \\
\hline Pain $(n=25)$ & $6.00(2.52)$ & $4.36(2.90)^{\mathrm{c}}$ & $3.68(3.09)^{\mathrm{a}}$ & $3.8(3.1)^{\mathrm{b}}$ & 7.37 & $0.235^{\star}$ \\
\hline Pain catastrophizing $(n=24)$ & $28.7(12.7)$ & $21.6(14.4)^{\mathrm{C}}$ & $16.0(12.3)^{\mathrm{a}, \mathrm{d}}$ & $17.8(13.7)^{\mathrm{b}}$ & 15.22 & $0.398^{*}$ \\
\hline Acceptance-AE $(n=24)$ & $19.6(7.19)$ & $26.2(8.31)^{C}$ & $30.6(9.05)^{\mathrm{a}}$ & $30.3(10.4)^{b}$ & 14.40 & $0.385^{\star}$ \\
\hline Acceptance-PW $(n=25)$ & $13.1(5.39)$ & $16.8(7.02)$ & $21.5(6.10)^{\mathrm{a}, \mathrm{d}}$ & $21.6(8.50)^{b}$ & 14.10 & $0.370^{\star}$ \\
\hline
\end{tabular}

Partial eta ${ }^{2}$ where 0.1 is a small effect, 0.25 is a medium effect, and 0.4 is a large effect. Variability of $\mathrm{n}$ is due to incomplete patient self-report data.

${ }^{*} P \leq 0.001$.

Using Bonferroni post hoc pair-wise comparisons:

${ }^{a}$ Significant change from pre to 3-month follow-up,

${ }^{\mathrm{b}}$ Significant change from pre to 6-month follow-up,

${ }^{\mathrm{C}}$ Significant change from pre to post,

${ }^{\mathrm{d}}$ Significant change from post to 3-month follow-up,

e Significant change from post to 6-month follow-up.

AE, activity engagement; AP, anticipated pain; FOPQ, Fear of Pain Questionnaire; PH, perceived harm; PHODA, photographs of daily activities; PW, pain willingness. 
This study has limitations. A potential limitation is the number of items selected for the daily diary. For practical reasons, only a few items assess each construct, and we consequently sacrificed comprehensiveness for brevity. However, our internal consistency estimates and outcomes from the full versions of the questionnaires do support our selections. In addition, we used a VAS scale for diary responses, blinding individuals to specific numerical values to decrease the likelihood of selecting a "favorite number." In an effort to enhance the likelihood of response variability, we administered the measures in a nonstandard format. Relatedly, the measures reported here are solely self-report, and they reflect only self-perceived changes. For future studies, inclusion of behavioral measures might strengthen the internal validity. In addition, a costeffectiveness analysis of GET Living will be critical for a wider dissemination and implementation of this treatment approach compared with the known costs and effectiveness associated with standard of care. The initial evidence in adults supports exposure's long-term cost effectiveness. ${ }^{9}$ Another limitation is that our diaries were delivered at the same time each day, which may have contributed to high completion rates, but may limit the sensitivity of measurement that ecological momentary assessment (EMA) would provide. EMAs are particularly useful when assessing mood and context, thus it is possible that fluctuations in mood or differing contexts may not have been fully captured with the same time daily approach. Future diary studies where items target mood and context changes would be particularly suited for EMA. Daily reporting of pain-related fears and catastrophic cognitions highly converged $(r=0.84)$ with changes in these 2 outcomes also aligned. From a clinical (and theoretical) perspective, these cognitions are likely best considered pain-related worries for youth, ${ }^{10}$ with the current results suggesting that separate evaluation of these 2 outcomes may be redundant.

Taken together, the findings in this study provide several suggestions for future work. This study represents the first application of SCEDs to evaluate exposure treatment in pediatric pain. This approach is ideally suited for this population given the highly specialized and complex nature of this participant group. The SCED approach allows for potentially evaluating mediators and moderators of treatment response without needing very large samples that are simply not feasible to recruit. This study also provides compelling evidence for GET for youth with chronic pain and elevated pain-related fear, supporting a precision medicine, screening-based tailored treatment approach. This model of interdisciplinary (psychology, PT) simultaneous treatment delivered in a pediatric pain outparticipant clinic is rarely implemented, and the findings in this study suggest its promise. Further research demonstrating the efficacy of this approach is warranted.

\section{Conflict of interest statement}

The authors have no conflicts of interest to declare.

\section{Acknowledgements}

The authors thank the additional clinicians involved in delivering GET Living (Ellen McGinnis, Cora Mukerji, Rupa Gambhir, Lauren Broccolo, Anne Norton, Erin Hung) and additional research coordinators in supporting the study implementation (Melissa Pielech, Molly Basch).

This investigation was supported by American Pain Society/ Sharon S. Keller Chronic Pain Research Grant, Deborah Munroe Noonan Memorial Research Fund, and NIAMS/R21 AR072921 awarded to L. Simons. J.W.S. Vlaeyen is supported by the research program "From acute aversive sensations to chronic bodily symptoms," a long-term structural Methusalem funding (METH/15/011) by the Flemish government, Belgium.

\section{Appendix A. Supplemental digital content}

Supplemental digital content associated with this article can be found online at http://links.Iww.com/PAIN/A898.

\section{Article history:}

Received 12 February 2019

Received in revised form 13 September 2019

Accepted 15 October 2019

Available online 28 October 2019

\section{References}

[1] Baek EK, Moeyaert M, Petit-Bois M, Beretvas SN, Van den Noortgate W, Ferron JM. The use of multilevel analysis for integrating single-case experimental design results within a study and across studies. Neuropsychol Rehabil 2014;24:590-606.

[2] Bailey KM, Carleton RN, Vlaeyen JW, Asmundson GJ. Treatments addressing pain-related fear and anxiety in patients with chronic musculoskeletal pain: a preliminary review. Cogn Behav Ther 2010;39:46-63.

[3] Bates D, Machler M, Bolker B, Walker S. Fitting linear mixed-effects models using Ime4. J Stat Softw 2015;67:1-48.

[4] Bergbom S, Boersma K, Linton SJ. Both early and late changes in psychological variables relate to treatment outcome for musculoskeletal pain patients at risk for disability. Behav Res Ther 2012;50:726-34.

[5] Crombez G, Bijttebier P, Eccleston C, Mascagni T, Mertens G, Goubert L, Verstraeten $\mathrm{K}$. The child version of the pain catastrophizing scale (PCSC): a preliminary validation. PAIN 2003;104:639-46.

[6] de Jong JR, Vlaeyen JW, Onghena P, Goossens ME, Geilen M, Mulder H. Fear of movement/(re)injury in chronic low back pain: education or exposure in vivo as mediator to fear reduction? Clin J Pain 2005;21:9-17; discussion 69-72.

[7] Dekker C, Goossens ME, Bastiaenen CH, Verbunt JA. Study protocol for a multicentre randomized controlled trial on effectiveness of an outpatient multimodal rehabilitation program for adolescents with chronic musculoskeletal pain (2B Active). BMC Musculoskelet Disord 2016;17: 317.

[8] den Hollander M, Goossens M, de Jong J, Ruijgrok J, Oosterhof J, Onghena P, Smeets R, Vlaeyen JW. Expose or protect? A randomized controlled trial of exposure in vivo vs pain-contingent treatment as usual in patients with complex regional pain syndrome type 1. PAIN 2016;157: 2318-29.

[9] den Hollander M, Heijnders N, de Jong JR, Vlaeyen JWS, Smeets R, Goossens M. Exposure in vivo versus pain-contingent physical therapy in complex regional pain syndrome type I: a cost-effectiveness analysis. Int $J$ Technol Assess Health Care 2018;34:400-9.

[10] Eccleston C, Fisher EA, Vervoort T, Crombez G. Worry and catastrophizing about pain in youth: a reappraisal. PAIN 2012;153: 1560-2.

[11] Flor $\mathrm{H}$. New developments in the understanding and management of persistent pain. Curr Opin Psychiatry 2012;25:109-13.

[12] Goubert L, Crombez G, Danneels L. The reluctance to generalize corrective experiences in chronic low back pain patients: a questionnaire study of dysfunctional cognitions. Behav Res Ther 2005;43:1055-67.

[13] Hayes SC, Hofmann SG, Stanton CE, Carpenter JK, Sanford BT, Curtiss $\mathrm{JE}$, Ciarrochi J. The role of the individual in the coming era of processbased therapy. Behav Res Ther 2019;117:40-53.

[14] I B, Onghena P. The single-case data analysis package analysing singlecase experiments with R software. J Mod Appl Stat 2013;12:450-78.

[15] Jamshidi L, Heyvaert M, Declercq L, Fernandez-Castilla B, Ferron JM, Moeyaert M, Beretvas SN, Onghena P, Van den Noortgate W. Methodological quality of meta-analyses of single-case experimental studies. Res Dev Disabil 2018;79:97-115.

[16] Kashikar-Zuck S, Flowers SR, Claar RL, Guite JW, Logan DE, LynchJordan AM, Palermo TM, Wilson AC. Clinical utility and validity of the Functional Disability Inventory among a multicenter sample of youth with chronic pain. PAIN 2011;152:1600-7.

[17] Knowles JE, Frederick C. merTools: tools for analyzing mixed effect regression models. R package version 030 2016. Available at: https:// rdrr.io/cran/merTools/. 
[18] Kuznetsova A, Brockhoff PB, Christensen RHB. ImerTest package: tests in linear mixed effects models. J Stat Softw 2017;82:1-26.

[19] Leeuw M, Houben RM, Severeijns R, Picavet HS, Schouten EG, Vlaeyen JW. Pain-related fear in low back pain: a prospective study in the general population. Eur J Pain 2007;11:256-66.

[20] Manolov R, Moeyaert M. How can single-case data Be analyzed? Software resources, tutorial, and reflections on analysis. Behav Modif 2017;41:179-228.

[21] Manolov R, Moeyaert M. Recommendations for choosing single-case data analytical techniques. Behav Ther 2017;48:97-114.

[22] McCracken LM, Gauntlett-Gilbert J, Eccleston C. Acceptance of pain in adolescents with chronic pain: validation of an adapted assessment instrument and preliminary correlation analyses. Eur J Pain;14:316-20.

[23] McGrath PJ, Walco GA, Turk DC, Dworkin RH, Brown MT, Davidson K, Eccleston C, Finley GA, Goldschneider K, Haverkos L, Hertz SH, Ljungman G, Palermo T, Rappaport BA, Rhodes T, Schechter N, Scott J, Sethna N, Svensson OK, Stinson J, von Baeyer CL, Walker L, Weisman S, White RE, Zajicek A, Zeltzer L. Core outcome domains and measures for pediatric acute and chronic/recurrent pain clinical trials: PedIMMPACT recommendations. J Pain 2008;9:771-83.

[24] Onghena P, Michiels B, Jamshidi L, Moeyaert M, Van den Noortgate W. One by one: accumulating evidence by using meta-analytical procedures for single-case experiments. Brain Impairment 2018;19:33-58.

[25] Palermo TM, Law EF, Zhou C, Holley AL, Logan D, Tai G. Trajectories of change during a randomized controlled trial of internet-delivered psychological treatment for adolescent chronic pain: how does change in pain and function relate? PAIN 2015;156:626-34.

[26] Parker RI, Vannest K. An improved effect size for single-case research: nonoverlap of all pairs. Behav Ther 2009;40:357-67.

[27] Parker RI, Vannest KJ, Davis JL. Effect size in single-case research: a review of nine nonoverlap techniques. Behav Modif 2011;35:303-22.

[28] Pielech M, Ryan M, Logan D, Kaczynski K, White MT, Simons LE. Pain catastrophizing in children with chronic pain and their parents: proposed clinical reference points and reexamination of the Pain Catastrophizing Scale measure. PAIN 2014;155:2360-7.

[29] Simons L, Kaczynski KJ, Conroy C, Logan D. Fear of pain in the context of intensive pain rehabilitation among children and adolescents with neuropathic pain: associations with treatment response. J Pain 2012;13:1151-61.

[30] Simons L, Pielech M, McAvoy S, Conroy C, Hogan M, Verbunt J, Goossens M. Photographs of Daily Activities (PHODA)-Youth English: validating a targeted assessment of worry and anticipated pain. PAIN 2017;158:912-21.

[31] Simons LE, Elman I, Borsook D. Psychological processing in chronic pain: a neural systems approach. Neurosci Biobehav Rev 2014;39: 61-78.

[32] Simons LE, Kaczynski KJ. The fear avoidance model of chronic pain: examination for pediatric application. J Pain 2012;13:827-35.

[33] Simons LE, Pielech M, Cappucci S, Lebel A. Fear of pain in pediatric headache. Cephalalgia 2015;35:36-44.

[34] Simons LE, Sieberg CB, Carpino E, Logan D, Berde C. The Fear of Pain Questionnaire (FOPQ): assessment of pain-related fear among children and adolescents with chronic pain. J Pain 2011;12:677-86.

[35] Simons LE, Sieberg CB, Conroy C, Randall ET, Shulman J, Borsook D, Berde C, Sethna NF, Logan DE. Children with chronic pain: response trajectories after intensive pain rehabilitation treatment. J Pain 2018;19:207-18.

[36] Simons LE, Sieberg CB, Pielech M, Conroy C, Logan DE. What does it take? Comparing intensive rehabilitation to outpatient treatment for children with significant pain-related disability. J Pediatr Psychol 2013;38: 213-23.

[37] Smeets RJ, Wittink H, Hidding A, Knottnerus JA. Do patients with chronic low back pain have a lower level of aerobic fitness than healthy controls?: are pain, disability, fear of injury, working status, or level of leisure time activity associated with the difference in aerobic fitness level? Spine 2006; 31:90-7; discussion 98.

[38] Tate RL, Perdices M, Rosenkoetter U, McDonald S, Togher L, Shadish W, Horner R, Kratochwill T, Barlow DH, Kazdin A, Sampson M, Shamseer L, Vohra $S$. The single-case reporting guideline in BEhavioural interventions (SCRIBE) 2016: explanation and elaboration. Arch Scientific Psychol 2016;4:10-31.

[39] Team R. R development core team. RA Lang Environ Stat Comput 2013; 55:275-86.

[40] Van Den Noortgate W, Onghena P. Multilevel Meta-analysis: a comparison with traditional meta-analytical procedures. Educ Psychol Measturement 2003;63:765-90.

[41] Van Den Noortgate W, Onghena P. A multilevel meta-analysis of singlesubject experimental design studies. Evidence-Based Commun Assess Interv 2008;2:142-51.

[42] Varni JW, Limbers CA, Burwinkle TM. Parent proxy-report of their children's health-related quality of life: an analysis of 13,878 parents' reliability and validity across age subgroups using the PedsQL 4.0 Generic Core Scales. Health Qual Life Outcomes 2007;5:2.

[43] Varni JW, Seid M, Kurtin PS. PedsQL 4.0: reliability and validity of the Pediatric Quality of Life Inventory version 4.0 generic core scales in healthy and patient populations. Med Care 2001;39:800-12.

[44] Varni JW, Seid M, Rode CA. The PedsQL: measurement model for the pediatric quality of life inventory. Med Care 1999;37:126-39.

[45] Vlaeyen J, Morley S, Linton SJ, Boersma K, de Jong J. Pain-related fear: exposure based treatment for chronic pain. Washington, D.C.: IASP Press, 2012.

[46] Vlaeyen JW, Crombez G, Linton SJ. The fear-avoidance model of pain. PAIN 2016;157:1588-9.

[47] Vlaeyen JW, de Jong J, Geilen M, Heuts PH, van Breukelen G. The treatment of fear of movement/(re)injury in chronic low back pain: further evidence on the effectiveness of exposure in vivo. Clin J Pain 2002;18: 251-61.

[48] von Baeyer CL. Children's self-reports of pain intensity: scale selection, limitations and interpretation. Pain Res Manag 2006;11:157-62.

[49] von Baeyer CL, Spagrud LJ, McCormick JC, Choo E, Neville K, Connelly MA. Three new datasets supporting use of the Numerical Rating Scale (NRS-11) for children's self-reports of pain intensity. PAIN 2009;143: 223-7.

[50] Walker LS, Greene JW. The functional disability inventory: measuring a neglected dimension of child health status. J Pediatr Psychol 1991;16: 39-58.

[51] Wicksell RK, Melin L, Lekander M, Olsson GL. Evaluating the effectiveness of exposure and acceptance strategies to improve functioning and quality of life in longstanding pediatric pain-a randomized controlled trial. PAIN 2009;141:248-57.

[52] Wiech K, Tracey I. The influence of negative emotions on pain: behavioral effects and neural mechanisms. Neuroimage 2009;47:987-94.

[53] Wiech K, Tracey I. Pain, decisions, and actions: a motivational perspective. Front Neurosci 2013;7:46.

[54] Zale EL, Lange KL, Fields SA, Ditre JW. The relation between pain-related fear and disability: a meta-analysis. J Pain 2013;14:1019-30. 